\title{
Salzmann nodular degeneration after photorefractive keratectomy for hyperopia
}

\author{
Pawan Prasher \\ Department of Ophthalmology, Sri Guru Ram Das Institute of Medical Sciences and \\ Research, Vallah, Amritsar, Punjab, India
}

\begin{abstract}
Purpose: To report a case of bilateral central Salzmann nodular degeneration (SND) after photorefractive keratectomy (PRK) for hyperopia.

Methods: Case report.

Results: A 47-year-old male presented with complaints of glare and difficulty with night driving. He had undergone PRK for hyperopia $(+3.5+1.5 \times 180$ in the right eye and $+4.5+1.0 \times 180$ in the left eye) in both eyes about 15 years back. The early postoperative period was uneventful; however, he started having these symptoms after two to three months. At presentation, uncorrected visual acuity was 20/100 and best corrected visual acuity was 20/30 with $+3.5+1.0 \times 180$ in both eyes. Slit-lamp examination showed bilateral 1-1.5 mm diameter, bluish white nodular lesions involving the central corneas. There were also prominent paracentral brown pigment ring deposits consistent with pseudo-Fleischer rings in both eyes. Scheimpflug images showed dense hyper-reflective nodules that were associated with elevated anterior corneal surface and measured $290 \mu \mathrm{m}$ in the right eye and $230 \mu \mathrm{m}$ in the left eye, along with presence of significant astigmatism (K1-45.1D, K2$47.8 D$ right eye and K1-45.4D, K2-49.2D left eye). Central pachymetry was $485 \mu \mathrm{m}$ in the right eye and $464 \mu \mathrm{m}$ in the left eye.
\end{abstract}

Conclusions: SND is a rare complication of PRK for hyperopia that can lead to suboptimal visual outcome. PRK should be included in the list of etiologies leading to SND.

Key words: Salzmann, degeneration, photorefractive keratectomy

\section{Introduction}

Salzmann nodular degeneration (SND) is a rare, non-inflammatory, degenerative condition of the cornea that is characterized by formation of usually bilateral, symmetrical, bluish-white elevated nodules on the anterior surface of cornea. ${ }^{1-3}$ The etiopathogenesis of this condition is still not clear, however, it is usually associated with chronic, recurrent corneal disorders like meibomian gland dysfunction, trachoma, phlyctenulosis, keratoconus, interstitial keratitis, vernal keratoconjunctivitis, measles, scarlet fever and other viral diseases..$^{3-5}$ SND has also been reported to develop after laser in situ keratomileusis (LASIK) surgery, pterygium surgery, cataract extraction, hard contact lens use and ocular trauma. ${ }^{4-7}$ Reported here, is a case that developed SND after photorefractive keratectomy (PRK) for hyperopia, a complication, which has not been previously reported in literature.

Correspondence: Dr. Pawan Prasher, Department of Ophthalmology, Sri Guru Ram Das Institute of Medical Sciences and Research, Vallah, Amritsar, Punjab, India.

E-mail: pawanprasher@yahoo.com 


\section{Case report}

A 45-year-old-male presented with complaints of glare and difficulty with night driving. He had undergone PRK for hyperopia in both eyes 15 years back elsewhere. Pre-op refraction was $+3.5+1.5 \times 180$ in his right eye and $+4.5+1.0 \times 180$ in his left eye. The details of the surgical procedure were not available. The immediate postoperative period was uneventful; however, he started having these symptoms after two to three months along with regression of his refractive error. The patient did not have any history of atopic disease or tendency for keloid formation and did not have any systemic illness. At presentation, uncorrected visual acuity was $20 / 100$ and best corrected visual acuity was 20/30 with $+3.5+1.0 \times 180$ in both eyes. Slit lamp examination showed 1-1.5 mm bluish-white nodules, one in each cornea, involving the central cornea with mild elevation on narrow slit illumination (Fig. 1). There was associated paracentral brown pigmented deposits, consistent with pseudo-Fleischer rings, in the corneal epithelium along with faint haze in the superficial paracentral stroma in both eyes. There was no sign of previous keratitis or associated blepharitis. Ocular surface staining test with fluorescein was negative. The remaining ocular and systemic examination was unremarkable. Scheimpflug images with Pentacam (Oculus, Wetzlar, Germany) showed dense, hyper-reflective nodules that were associated with elevated anterior corneal surface and measured $290 \mu \mathrm{m}$ in the right eye and $230 \mu \mathrm{m}$ in the left eye (Fig. 2). Sagittal sections showed irregular steepening of central corneas in both eyes with significant astigmatism (K1- 45.1D, K2- 47.8D right eye and K1- 45.4D, K2- 49.2D left eye). The corneal thickness at the center of the pupil was $485 \mu \mathrm{m}$ in the right eye and $464 \mu \mathrm{m}$ in the left eye. The patient was counselled about surgical options including superficial keratectomy with application of mitomycin $\mathrm{C}$ and phototherapeutic keratectomy; however, he refused any surgical intervention.
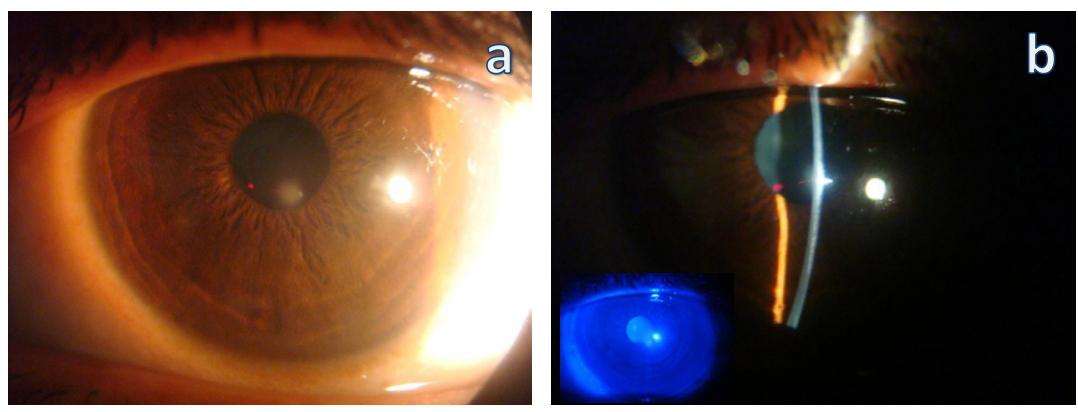

Fig. 1. Slit-lamp picture of left eye shows bluish-white nodular opacity in the papillary area (a), with anterior surface elevation on narrow slit beam illumination (b). Inset (b, bottom left) shows paracentral pseudo-Fleischer ring under cobalt blue filter illumination. 

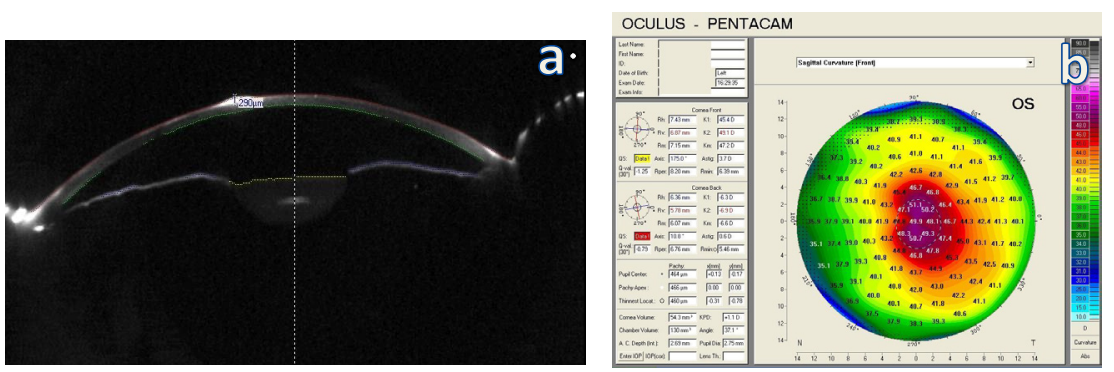

Fig. 2. Scheimpflug image of left eye shows dense hyper reflective nodular lesion measuring 290 $\mu \mathrm{m}$ (a). Sagittal section shows irregular steepening in the central part of cornea corresponding with the site of the nodule (b).

\section{Discussion}

PRK has been found to be a safe and predictable procedure for the correction of mild to moderate hyperopia and involves steepening of the central curvature by flattening the peripheral curvature by laser correction. ${ }^{8-9}$ Common complications of this procedure include loss of corneal transparency due to haze formation, refractive error regression, and formation of surface irregularities..$^{10}$ Formation of central corneal haze, a well-known complication after PRK for myopia, is not seen in hyperopes. O'Brart et al. did not see loss of central corneal transparency in any of the 40 eyes with 7.5-years follow-up. ${ }^{9}$ However, they reported peripheral ring of haze and sub-epithelial iron ring deposition, which was present in our case too. Although we could not find any previous report of SND after PRK, we believe it might be a case of using different nomenclature to describe this condition. Stakheev et al. reported one case of bilateral apical nodular sub-epithelial scar leading to irregular astigmatism in their study of 98 eyes of 52 patients who underwent PRK for hyperopia. ${ }^{8}$ Although the clinical picture of our patient is similar to this case, a definite comparison could not be made as photograph of that case has not been published. Sener et al. reported 12 eyes of six patients with apical sub-epithelial nodular scars in eyes that had hyperopic PRK retreatment. ${ }^{11}$ Comparison of their published photographs shows similarity with the current case. It is interesting that these nodular opacities were not labelled as SND. The reason for this could be lack of definite distinguishing features between these two entities in ophthalmic literature.

SND is usually considered an idiopathic condition associated with chronic inflammatory ocular surface diseases. However, it has also been reported after refractive surgeries like LASIK. ${ }^{4-6}$ The pathogenesis of SND is not clear; however, previous authors have speculated that these nodules develop from chronic ocular surface irritation, uneven surfaces, corneal exposure and secondary to tear film disturbances. $^{5-6}$ In their cases of SND after LASIK, the patients showed clinical features of dry eye in the postoperative period. Although our patient did not show any evidence of dry eyes at presentation, its presence in early postoperative period cannot be ruled out. We speculate that SND in the current case could be the result 
of aberrant wound healing response of the cornea to the surgical trauma. Interestingly, the central corneal thickness of our case was towards the lower side, but its role in SND formation could not be established. It can be argued that the current case could be labelled as a hypertrophic scar and not SND. However, ophthalmic literature is not very clear on distinction between these two entities. We believe these entities have strong analogy to dermatological conditions of keloid and hypertrophic scar. However, in dermatology literature, keloids and hypertrophic scars have been shown to be having different characteristics on histology and immunochemistry. ${ }^{12} \mathrm{~A}$ similar study on human corneas can provide further insight into this pathology. At present, the diagnosis of SND is clinical due to wide variation in the pathology of these nodules. ${ }^{13-14}$ Various imaging modalities like optical coherence tomography (OCT) have proved to be helpful in the in-vivo analysis of the morphological characteristics of this pathology. ${ }^{6-7}$ Scheimpflug images of the current case revealed nodular elevation of the anterior surface, significant astigmatism and allowed measurement of the nodules, which shows that it can be a useful imaging modality in analysis of this pathology. Although we could not perform histopathology on the current case, previous studies have shown a strong correlation with OCT findings.?

The conservative medical management of SND includes topical lubricants, warm compresses ocular hygiene, topical non-steroidal anti-inflammatory therapy, topical steroids and oral doxycycline. ${ }^{1}$ However, these topical and systemic measures are unsatisfactory in achieving cure of the nodules and surgical treatment may be indicated in symptomatic cases. Various surgical modalities described in literature for treatment of SND include manual removal or nodulectomy with or without topical mitomycin $C$ application, superficial keratectomy with or without amniotic membrane transplantation, nodulectomy followed by excimer laser phototherapeutic keratectomy to smoothen the corneal surface, cryotherapy and lamellar keratoplasty or penetrating keraoplasty. ${ }^{13,15}$ Although our patient was counselled about these options, he did not want any surgical intervention at the time.

\section{Conclusion}

SND is a rare complication of PRK for hyperopia that can lead to suboptimal visual outcome. PRK should be included in the list of etiologies leading to SND.

\section{References}

1. Graue-Hernández EO, Mannis MJ, Eliasieh K, Greasby TA, Beckett LA, Bradley JC, Schwab IR. Salzmann nodular degeneration. Cornea 2010;29:283-289.

2. Roszkowska AM, Aragona P, Spinella R, Pisani A, Puzzolo D, Micali A. Morphologic and confocal investigation on Salzmann nodular degeneration of the cornea. Invest Ophthalmol Vis Sci 2011;52:5910-5919.

3. Farjo AA, Halperin GI, Syed N, Sutphin JE, Wagoner MD. Salzmann's nodular corneal degeneration clinical characteristics and surgical outcomes. Cornea 2006;25:11-15.

4. Lim MC, Chan WK. Salzmann nodular degeneration after laser in situ keratomileusis. Cornea 2009;28:577-578. 
5. Moshirfar M, Chang JC, Mamalis N. Salzmann nodular degeneration after laser in situ keratomileusis. Cornea 2010;29:840-841.

6. VanderBeek BL, Silverman RH, Starr CE. Bilateral Salzmann-like nodular corneal degeneration after laser in situ keratomileusis imaged with anterior segment optical coherence tomography and high-frequency ultrasound biomicroscopy. J Cataract Refract Surg 2009;35:785-787.

7. Hurmeric V, Yoo SH, Karp CL, Galor A, Vajzovic L, Wang J, Dubovy SR, Forster RK. In vivo morphologic characteristics of Salzmann nodular degeneration with ultra-high resolution optical coherence tomography. Am J Ophthalmol 2011;151:248-256.

8. Stakheev A. Excimer laser photorefractive keratectomy in hyperopia. Asian J Ophthalmol 2000;2:7-10.

9. O'Brart DP, Patsoura E, Jaycock P, Rajan M, Marshall J. Excimer laser photorefractive keratectomy for hyperopia: 7.5-year follow-up. J Cataract Refract Surg 2005;31:1104-1113.

10. Spadea $L$, Verrecchia V. Effectiveness of scraping and mitomycin $C$ to treat haze after myopic photorefractive keratectomy. Open Ophthalmol J 2011;5:63-65.

11. Sener B, Ozdamar A, Aras C. Apical nodular subepithelial corneal scar after retreatment in hyperopic photorefractive keratectomy. J Cataract Refract Surg 2000;26:352-357.

12. Ehrlich HP, Desmoulière A, Diegelmann RF, Cohen IK, Compton CC, Garner WL, Kapanci Y, Gabbiani G. Morphological and immunochemical differences between keloid and hypertrophic scar. Am J Pathol 1994;145:105-113.

13. Werner LP, Issid K, Werner LP, Pouliquen Y, Legeais JM, Renard G. Salzmann's corneal degeneration associated with epithelial basement membrane dystrophy. Cornea 2000;19:121-123.

14. Obata $H$, Inoki T, Tsuru T. Identification of oxytalan fibers in Salzmann's nodular degeneration. Cornea 2006;25:586-589.

15. Das S, Link B, Seitz B. Salzmann's nodular degeneration of the cornea: a review and case series. Cornea 2005;24:772-777. 